\title{
Utilidad de la procalcitonina para valorar la necesidad de cirugía y la presencia de isquemia y necrosis en pacientes con obstrucción del intestino delgado
}

\author{
Usefulness of procalcitonin to assess the need for surgery and the presence of ischemia \\ and necrosis in patients with small bowel obstruction
}

\author{
Ansony R. Godínez-Vidal1*, Guillermo E. Hernández-Rodríguez¹ y Sandra C. López-Romero² \\ ${ }^{1}$ Departamento de Cirugía General; ${ }^{2}$ Clínica de Sepsis. Hospital General de México Dr. Eduardo Liceaga, Ciudad de México, México
}

Al editor,

He leído con gran interés el artículo original de Evaristo-Méndez, et al. ${ }^{1}$ publicado en Cirugía y Cirujanos. Este trabajo refleja el ánimo de los cirujanos de sustentar la necesidad de intervención quirúrgica en pacientes con obstrucción del intestino delgado.

Queremos felicitar a los autores por el entusiasmo de realizar un estudio prospectivo de alto valor científico para beneficio de la atención a los pacientes.

Sus resultados son sumamente interesantes. Por la fisiología de la procalcitonina creemos que en el estudio es necesario excluir a pacientes portadores de patologías crónicas susceptibles de modificar la respuesta inflamatoria-inmunitaria, antecedentes de cáncer, inmunosupresión, insuficiencias orgánicas crónicas, toxicomanías, tratamiento previo con quimioterapia o radioterapia, internamiento posoperatorio menor de 24 horas, o que hayan sido sometidos a procedimiento quirúrgico por cualquier otra causa en un periodo menor de 90 días.

En un estudio multicéntrico que incluyó 128 pacientes con diagnóstico de colitis isquémica e infarto mesentérico se reportaron valores más altos de procalcitonina en aquellos con necrosis y que fallecieron, concluyendo que la procalcitonina se puede utilizar como un marcador de necrosis ${ }^{2}$. Uno de los metaanálisis más grandes reportados en la literatura mundial incluye cinco estudios y un total de 659 pacientes, y reporta que la sensibilidad para la detección de isquemia intestinal osciló entre el $72 \%$ y el $100 \%$, y la especificidad entre el $68 \%$ y el $91.3 \%$, concluyendo que la procalcitonina se puede utilizar en la práctica diaria como una herramienta para diagnosticar la isquemia intestinal ${ }^{3}$.

Un buen marcador debe servir para el diagnóstico de una patología y para valorar la evolución de esta, es decir, como factor pronóstico. Bajo esta premisa no existe actualmente un marcador que cumpla al cien por ciento con dichas características. En términos quirúrgicos se traduce en un retraso de la exploración quirúrgica y un aumento de la mortalidad. Por ello, como los mismos autores del trabajo reconocen, sí vale la pena continuar realizando estudios adicionales para reafirmar o invalidar los hallazgos presentados.

Enhorabuena por el citado trabajo, ya que es de gran complejidad tomar la decisión de reoperar y existen pocos artículos prospectivos que estudien la posible presencia de isquemia 0 de necrosis en la obstrucción mecánica aguda del intestino delgado.

\footnotetext{
Correspondencia:

*Ansony R. Godínez-Vidal

Dr. Balmis, 148

Col. Doctores, Del. Cuauhtémoc

C.P. 06726, Ciudad de México, México

E-mail: ansony.rgv@gmail.com

Fecha de recepción: 08-06-2019

Fecha de aceptación: 30-03-2020

DOI: 10.24875/CIRU.20001351

Cir Cir. 2020;88(4):535-536

Contents available at PubMed

www.cirugiaycirujanos.com

0009-7411/@ 2020 Academia Mexicana de Cirugía. Publicado por Permanyer. Este es un artículo open access bajo la licencia CC BY-NC-ND (http://creativecommons.org/licenses/by-nc-nd/4.0/).
} 


\section{Financiamiento}

Los autores declaran que no se recibió ningún tipo de financiamiento para la redacción de la presente carta al editor.

\section{Conflicto de intereses}

Los autores declaran no tener conflictos de intereses.

\section{Bibliografia}

1. Evaristo-Méndez G, Gallegos-Sierra C, Cruz-Temores S. Valor predictivo de la procalcitonina para la necesidad de cirugía y la presencia de isquemia y necrosis en pacientes con obstrucción del intestino delgado. Cir Cir. 2019;87:45-52.

2. Cosse C, Sabbagh C, Browet F, Mauvais F, Rebibo L, Zogheib E, et al. Serum value of procalcitonin as a marker of intestinal damages: type, extension, and prognosis. Surg Endosc. 2015;29:3132-9.

3. Cosse C, Sabbagh C, Kamel S, Galmiche A, Regimbeau JM. Procalcitonin and intestinal ischemia: a review of the literature. World J Gastroenterol. 2014;20:17773-8. 